\title{
MODELOS INFANTILES Y MODELOS CIENTÍFICOS SOBRE LA MORFOLOGÍA DE LOS SERES VIVOS
}

\author{
ARCÁ, M. y GUIDONI, P. \\ Centro di Studio degli Acidi Nucleici. Roma. \\ Seminario Didattico. Facoltà di Scienze. Nápoles.
}

Traducción de M.P. Jiménez Aleixandre.

\section{SUMMARY}

This work deals with chrildren's models and scientific models on morphology and physicology of living beings.

\section{QUÉ ES UN MODELO Y POR QUÉ TRA- BAJAR CON MODELOS}

La misma palabra modelo tiene, entre nosotros, una ambigua multiplicidad de significados (Bachelard 1979).

Por un lado puede significar el original, el arquetipo al que nos referimos y que consideramos ejemplar, en este sentido podemos tomar una determinada cosa como modelo para otra que queremos construir; o podemos tomar un determinado proceso (o situación) emblemático como modelo para otros procesos o situaciones que tratamos de reproducir o de hacer suceder.

Por otra parte, modelo puede significar una esquematización precisa que construimos sobre la base de una multiplicidad de datos de la experiencia (lo que llamamos a veces datos de la realidad), que da lugar a una abstracción satisfactoria de como «funcionan» las cosas.

Modelo puede ser también el prototipo de una clase, a lo que hacemos referencia por medio del lenguaje y de la imaginación para reconocer rasgos similares en objetos, hechos, procesos o situaciones, con el objetivo de agruparlos en la clase identificada por el prototipo.

Por último, con modelo también podemos referimos a estructuras hipotéticas, probables, de la realidad, inaccesibles a la evidencia directa, cuyos rasgos se suponen responsables de las correlaciones mutuas entre fenómenos observados.

En las observaciones sobre actividades cognitivas, vemos que todos los significados incluidos en una misma palabra son difícilmente separables entre sí; del mismo modo distintas palabras pueden expresar rasgos especificos de significados parecidos, realzados de dis- tinta forma en diferentes contextos; distinguimos entre modelos y esquemas, reconstrucciones abstractas y representaciones de la realidad.

De todas formas un modelo no es visto como una cosa ni como un objeto con su propia concreción. Un modelo, en su naturaleza de estructura de relación, pertenece a un dominio «lingüístico" (Bachelard 1979), in cluso cuando somos capaces de materializarlo en un objeto: opera de hecho como un filtro intermedio en el que delegamos parte de nuestras funciones de conocimiento. A veces un modelo actúa como una visualización de rasgos «abstractos", relacionando en un mar. co espacio-temporal acontecimientos singulares; u organiza en secuencias dinámicas pautas de la realidad que no podemos conseguir sin su ayuda. Pero en ningún caso abarca ni representa todas las caracteristicas de la realidad a que se refiere.

El objetivo general de un modelo es pues reducir (restringir) la cantidad de lo que es aún desconocido en un campo no del todo conocido, y permitir a los elementos de lo que se conoce coagular en una forma determinada y compleja. En todo caso, un modelo es un poderoso instrumento mental, especialmente apto para la comprensión de estructuras de la realidad, cuando su complejidad no nos permite alcanzar y representar directamente sus múltiples relaciones de conexión, y también para lograr un control directo del significado de los hechos.

La actividad cognitiva de modelado tiene lugar en paralelo a la de construcción de esquemas (Arcà et al. 1984), capaz de identificar y seleccionar datos de la experiencia de acuerdo con alguna pauta común, consistente con las estructuras de propósito e interpretación. 
En esta perspectiva, cualquier actividad de elaboración de modelos discrimina elementos pertinentes, y después los reorganiza en un «todo» esquemático, cuyos ras" gos pueden corresponderse con la situación problemá. tica dada -elegida - a la que uno se enfrenta. En estos casos, sin embargo, el modelo no actúa como un copista neutral de la realidad, sino más bien como un agente selectivo (filtro y soporte al mismo tiempo) sobre las características de la realidad, preparado para una identificación abstracta y completa de aspectos específicos de la propia realidad.

Pero al mismo tiempo que un modelo aparta la aten. ción (abstrae) de muchas características de la realidad, también aporta a la reconstrucción organizadora mu* chos rasgos nuevos, que pueden no encontrar correspondencia directa con la realidad a partir de la cual comenzo la actividad de modelado. En este sentido un modelo siempre comporta su propia originalidad, en cuanto añade a la selección esquemática de los hechos observados otros trazos peculiares pertenecientes a su propia naturaleza de modelo. Por tanto las actividades de construcción de modelos siempre llevan a cabo una profunda transformación de la realidad, tanto empobreciéndola por medio de esquematizaciones, como enriqueciéndola a través de reconstrucciones «adecua das", y el mismo modelo se convierte en un polo activo del proceso cognitivo, estimulando la búsqueda y la explicación de rasgos concretos de la realidad, no observados previamente, o que parecían irrelevantes (esta dinámica de bucles entre la realidad y sus mode los está bien documentada tanto en la evolución histórica como en el desarrollo individual del conocimiento),

\section{CONOCIMIENTO POR MEDIO DE MODELOS}

Tanto en las construcciones cognitivas de los individuos, como en las construcciones culturales realizadas a lo largo del tiempo por las sociedades humanas, to do conocimiento del mundo es en realidad conocimien. to de distintas series de modelos del mundo. En su desarrollo cultural, el conocimiento científico humano ha producido, sucesiva y alternativamente modelos progresivamente más adecuados para explicar y para permitir la representación de aspectos más y más comple. jos de la realidad. En el desarrollo individual, el niro se dedica a la organización, tanto sucesiva como alternativa, de modelos progresivamente más adecuados pa. ra representar aspectos específicos y generales de su experiencia del mundo.

Con pautas similares, todo conocimiento individual cre ce conformado por los hechos de la realidad y por las culturas ambientales, común y científica, que lo rodean, intentando explicar fenomenos naturales a varios niveles de comprensión y expresión; intentando conectarlos entre si de forma lógica (es decir a semejanza de un modelo) por medio de representaciones complejas; intentando explicar las estructuras internas que se pue - den suponer o reconocer, a veces, en fenómenos y hechos.

En todo caso, cuanto más apto es el modelo para referirse a la "verdadera» realidad (que sucede), con mayor eficiencia puede predecir cómo tendrán lugar las cosas. (Modelado e inferencia son aspectos indispensables e inseparables de cualquier construcción cognitiva o cientifica). $Y$ las predicciones acerca de la experiencia futura, pueden siempre confrontarse con experiencias reales que sucederán a continuación, en el interminable juego de reforzar, modificar y adecuar el modelo original a la realidad efectiva. Esta actividad de confrontación sistemática que discurre por medio del error, la corrección y la invención, por aproximaciones sucesivas, define una forma general de construcción del conocimiento que parte de lo conocido, pero que se dedica continuamente a readaptar los conocimientos viejos a los nuevos.

Por otro lado, cualquier comprensión nueva parte de lo que conocemos; sin embargo, no sólo de lo que percibimos directamente acerca de nosotros mismos y de la realidad en torno nuestro, sino también de lo que indirectamente sabemos a través de la cultura que nos rodea. Vivimos en un medio tanto natural como cultural, y desde el nacimiento experimentamos ambos como adecuados el uno al otro, de forma que podemos utilizar ambas fuentes de experiencia cognitiva como bases para la construcción de conocimientos personales nuevos. Comenzamos así nuestro desarrollo cognitivo partiendo de series o familias de observaciones, nociones, frases, explicaciones, significados, pero pronto nos vemos obligados a darle una estructura a tal variedad (cuantitativa y cualitativa) de nociones y obser. vaciones, con objeto de adecuarlas mejor a la realidad cambiante y de establecer conexiones recíprocas. Es difícil poseer - a priori- marcos (esquemas) cognitivos -iun modelo de modelos! - capaces de organizar de forma consistente nociones y observaciones de áreas específicas de la realidad; y debemos definir, al mis. mo tiempo, modelos locales y esquemas generales pa. ra sostenerlos. Al hacer eso, sobreimponemos a hechos que experimentamos diversas representaciones esquemáticas de la realidad, las conectamos continuamente para elaborar modelos. Guiados por sugerencias de los que ya existen, comparamos modelos particulares y específicos, establecemos analogías experimentales y cognitivas entre ellos, intentamos combinar algunos para organizar otros más inclusivos (Guidoni 1985).

Con frecuencia, explicar la construcción de estructuras complejas a partir de otras más simples da lugar a paradojas (Bareiter 1985), sin embargo, una de las estrategias cognitivas que parece ser capaz de «llegar a más partiendo de menos» es la anaiógica (Guidoni 1985, Bareiter 1985). Analogia, en sentido amplio, sig. nifica una semejanza formal parcial entre dos (o más) representaciones de aspectos de la realidad, ayudando uno de ellos, el relativo a aspectos mejor conocidos, al otro, en su tarea específica de organizar y repre- 
sentar un conjunto distinto de aspectos. Los modelos analogicos son bastante comunes; sin embargo, la estrategia cognitiva basada en procesos de analogía nos ofrece la posibilidad de confrontar modelos entre sí, de confrontar situaciones reales entre sí a través de sus modelos y, finalmente, de construir modelos nuevos, más complejos (o más simplificados), con los que comprender y reinterpretar los iniciales.

\section{PRIMEROS MODELOS}

En la escuela infantil (Arcà et al. 1988), niños de 4 años están ocupados en responder a una cuestión sugestiva, están tratando de dar forma y representar sus «modelos de ver». En una conversación dirigida por el maestro, cada niño sugiere su propio argumento, expresando en voz alta la forma en que imagina que se "ve», y cada uno tiene ideas muy personales sobre el tema. El problema fundamental es siempre el mismo, tanto para los griegos clásicos como para los nirios de hoy dia, ¿Como puede el ojo «capturar» algo de la realidad externa y meterlo en las estructuras internas del «yo»? ¿De qué forma alguna entidad interna -el cerebro, la mente- «se apodera» del mundo exterior? Hay quien sugiere, como nuestros niños, unos polvos mágicos de visión. Al final $\rightarrow$ como resultado- muchos niftos se ponen de acuerdo en una forma común de representar la «vista» por un dibujo: un gran ojo, con sus pestañas, iris y pupila... y dentro del círculo de la pupila el ojo contiene la imagen coloreada de los objetos vistos, una escena pintada del mundo que lo rodea.

Dibujando imágenes vistas como sobreimpuestas a ojos abiertos, los nif̆os explícitamente (incluso si es de forma inconsciente) modelan la relación que conecta siempre, por medio del acto visual, el interior del cuerpo con su exterior; en realidad representan el rasgo básico del acto de ver, la "captura» visual de lo que uno está viendo como un fragmento de la realidad. Así, mientras podemos observar que la construcción de modelos acerca de las actividades físiológicas se desarrolla precozmente en la evolución cognitiva de los niffos (Arcà et al. 1988), también constatamos que cualquier modelado ingenuo de las percepciones, por más naïf que pueda parecer, se esfuerza por expresar de forma significativa las conexiones esenciales entre los individuos y el entorno, que toman forma por la adecuación de percepciones específicas a rasgos específicos del mundo.

\section{MODELOS INFANTILES (Giordan et al. 1983)}

En una situación normal de clase, niños (7 años) discuten entre ellos y con su profesor; al comenzar actividades nuevas están acostumbrados a confrontar sus opiniones personales sobre el tema escogido, como punto de partida para las observaciones y experiencias que seguirán. Ahora están discutiendo acerca del funcionamiento del ojo y de la percepción visual relacionada con él. Cada niño se dedica a dar una explicación inicial de lo que pasa cuando está viendo el objeto particular que tiene delante. ¿Como puede uno (un ser humano) ver algo? ¿Qué procesos mentales, qué correlaciones, qué rasgos físicos están conectados de forma imprescindible en un fenómeno tan asombroso? Las cosas están ahí, pero si uno las ve uentran» en el ojo de uno, o en el cerebro de uno... no el objeto mismo, sino una imagen del objeto, una fotografia del objeto; por supuesto el ojo es como una cámara de fotos... Pero pensamos con el cerebro, y es el cerebro el que da "órdenes» al ojo: el cerebro le ordena al ojo «ver»... el ojo hace la foto y el cerebro actúa como una máquina de revelado, y ve la foto... y nosotros vemos el objeto. Está claro.

En estas discusiones, vemos como los modelos analógicos actúan para explicar procesos desconocidos. El desarrollo coherente de analogías de primer orden es utilizado por los ninos para explicar el funcionamiento del proceso en conjunto. Los procedimientos de elaboración de modelos siempre tratan de conectar la experiencia directa de los niños con la reelaboración de pautas culturales transmitidas del conocimiento de los adultos al de los niños a través de canales lingüisticos. Entonces, la cuestión para el profesor es: ¿Qué contenido de realidad dan los niños a sus propios modelos? En otras palabras: ¿Cuál es el significado - por ejemplo en esta situación- del «parecido» evocado por los nif̂os entre un ojo y una cámara, ya que es claramente imposible pensar en una concreción física de máqui. nas de fotografiado y revelado en el cerebro? ¿Cómo puede entonces un adulto, un profesor, introducirse en el significado adscrito a la multiplicidad de analogías (es como...) que los niffos con frecuencia producen y manejan en sus explicaciones de algunos aspectos -inexplicables de otra forma-- de la realidad?

\section{REPRESENTANDO MODELOS}

En resumen, en la multiplicidad de reconstrucciones cognitivas de los sucesos por los niños, podemos reconocer muchas dimensiones explicativas realzadas a través de sistemas específicos de elaboración de modelos. Cada modelo infantil de la realidad suele estar enfocado hacia una forma definida de observar una situa. ción compleja, apropiada para explicarla parcialmente; mediante un modelo el niño es capaz de interpretar parcialmente hechos y fenomenos de acuerdo con un punto de vista muy especifico, y la correspondiente conciencia metacognitiva se desarrolla de forma bastante temprana (Mazzoli 1987). Así, podemos reconocer modelos espaciales en los que las relaciones entre sistemas o entre partes de un conjunto están expresadas en términos de su organización espacial; las características en las que se pone énfasis son, por tanto, fundamentalmente formas, posiciones y localizaciones. Un modelo "correcto» de este tipo explica cómo y dónde es- 
tán situados los objetos, o sus partes, en un esquema dado; incluyendo a veces, por la misma organización topológica del espacio, algunas de las relaciones entre sistemas, o entre sus partes. La mayoría de los modelos ingenuos sobre el cuerpo humano pertenecen a este tipo espacial (Giordan 1983, 1987), y, en nuestra experiencia los hemos encontrado muy similares en niños de 4 y 12 años. No resulta sorprendente que estas representaciones no se modifiquen sustancialmente con la edad: a menudo, durante este intervalo, a los niffos se les pide soblo que memoricen nombres y posiciones de partes distintas, sin ninguna sugestión de nuevas dimensiones potenciales del conocimiento aptas para suscitar y apoyar modelizaciones más complejas. (En muchos libros de texto la única connotación de organos y aparatos es por su forma y posición dentro de un cuerpo considerado como un contenedor de organos).

En otros casos podemos reconocer modelos temporales y el espacio de la representación es usado a menudo con un significado temporal. Los nintos tratan así de representar las sucesiones temporales de acontecimientos por medio de múltiples dibujos distintos de! mismo objeto, sistema o situacion, como hacen con frecuencia en el caso de objetos que se mueven por secuencias de posición (Mazzoli 1987). Y, a menudo, resulta dificil para un adulto interpretar la rica variedad de formas, apropiadas para representar y elaborar modelos de secuencias temporales, con que los niffos son normalmente capaces de operar. El problema es doble: por un lado cómo transformar, mediante procesos de representación, la comprensión de una secuencia diacrónica en una forma sincrónica esquemática; por otro lado, cómo hacer converger en una evolución global, temporal, los distintos aspectos de un funcionamiento que necesitan ser interpretados -modeloscomo funcionamiento en paralelo, a menudo por interacción y causación recíproca.

A veces los modelos de los alumnos pueden corresponder a una exigencia de conectar, en una dimensión espacio-temporal, varios aspectos funcionales de un sistema, independientes pero correlacionados. Aparecen entonces problemas de simbolización y comunicacion, en relación a modelos funcionales: el primero, por ejemplo es la forma de representar la función misma, el propio acto de hacer algo de una forma muy específica, y no sólo la forma de las estructuras responsables; el segundo la forma de elaborar modelos de una multiplicidad de funciones, cada una de ellas funcionando con sus tiempos y ritmos, en un funcionamiento ordenadamente orgánico, etc.

La elaboración de modelos de funciones aparece como una tarea muy difícil; sin embargo, por ejemplo cualquier conocimiento factual (realista) de los sistemas vivientes tiene que estar fundado en la reorganización cognitiva de los procesos bioquímicos parciales, en esquemas de funcionamiento muy dinámicos. Sólo un modelo complejo de la respiración, absorción y digestión, difusión y circulación, excreción... a nivel tanto celular como de organismo, puede explicar los rasgos generales de la vida. No hay problemas, hasta que este tipo de elaboración de modelos corresponde a reconstrucciones mentales ocultas; no es imposible pensar acerca de procesos dinámicos, acerca de cambios y transformaciones en el tiempo. Pero ¿qué pasa, si por ejemplo en una clase de ciclo superior, se pide explícitamente a los niños que expresen y representen un modelo personal dinámico de los procesos de la digestion humana? Las actividades fisiologicas tienen lugar de acuerdo con pautas obligadas, y cualquier elaboración de modelos de ciencias, apoyada en esquemas de interpretación causal, tiene que ser coherente con la forma en como suceden los hechos. Incluso la forma de elaboración de modelos por parte de los alumnos no puede eludir las conexiones logicas (causales) dentro de un proceso consistente (inluso cuando es desconocido); por tanto es casi imposible inventar un modelo ficticio sustancialmente diferente del «correcto". Los alumnos, por tanto, prueban a inventar maquinarias estrambóticas para realizar las pautas, obligatoriamente complejas, de una actividad digestiva; o atribuyen nombres inventados a las partes y estructuras en acción, esenciales, de acuerdo con sus esquemas, para cumplir papeles indispensables. Como resultado, incluso los modelos digestivos más personalizados, a menudo reproducen, por sus estructuras internas, rasgos conocidos de tipo convencional (científico), también en los casos en que esto conduce a imágenes estáticas de formas $y$ de redes de formas.

\section{INTERPRETANDO MODELOS}

Tanto en el pensamiento científico como en el infantil, la explicación causal de los acontecimientos de la realidad por reconstrucción cognitiva está a menudo fundada en hipotesis de modelos subestructurales: de pequerlas estructuras «materiales» (microscópicas, submicroscópicas, atómicas) (Rimondi 1988) capaces de explicar rasgos macroscópicos de hechos y fenómenos. En los sistemas biológicos, las células microscópicas pueden (se supone que lo hacen) unirse para formar tejidos u órganos, en tanto que las partículas submicroscópicas se pueden agrupar para dar las estructuras celulares, y el "funcionamiento» de las partículas submicroscópicas puede ser visto como responsable del funcionamiento de la célula en conjunto... En los sistemas químicos, los rasgos macroscópicos de la materia y las transformaciones dependen de los rasgos y la reactividad de los átomos y moléculas en los sistemas físicos, etc.

Pero en el pensamiento infantil, Ia elaboración de modelos microscópicos plantea cuestiones muy profundas ¿qué clase de propiedades macroscópicas son causadas por cuáles propiedades de las particulas submicroscopicas? ¿Posee una simple «molécula» de un material dado las propiedades macroscópicas del material? ¿Puede una molécula de oro ser dorada o dúctil?... y, 
si no es así. ¿Cómo explicar las propiedades «áureas»? Enfrentándose a estas cuestiones imposibles de eludir ¿Cómo puede un profesor convencer a sus alumnos de que las propiedades de los materiales se pueden correlacionar con la organización colectiva de las moléculas que individualmente no comparten ninguna de las propiedades consideradas?

Problemas similares se suscitan en el manejo de modelos experimentales. Está claro para un profesor, tanto como para un científico, que cualquier experimento corresponde a un modelo ésquemático de sucesos que tiene como fin reconstruir sólo algunos aspectos destacados de pautas más complejas. Muchas cuestiones planteadas frente al suceder auténtico de acontecimientos demandan respuestas experimentales; todo experimento es un acontecimiento reconstruido imitando la realidad, pero los montajes específicos, lectura de resultados e interpretacion de datos, siempre implican modelos abstractos a priori del fenómeno real como conjunto. Guiados por el modelo, los resultados de las reconstrucciones «experimentales» pueden ocupar su lugar otra vez en los contextos "naturales», de acuerdo con una especie de sobreimposición de reconstrucciones esquemáticas artificiales de aspectos de la realidad a la propia realidad como conjunto. Con demasiada frecuencia, para los nirtos, un resultado experimental es sólo el fin de un proceso, y no reconocen en las operaciones experimentales las pautas esquematizadas de un suceso real.

En la escuela, cuando pretendemos enseñar a los nihos «a ver» relaciones sencillas entre los rasgos morfológicos y fisiológicos de los organismos, les encargamos la tarea de reconocer partes anatómicas de los animales, y, al mismo tiempo les sugerimos modelos mecánicos de funciones fisiologicas, en las que objetos mecánicos imitan, a su nivel, organos o estructuras corporales responsables de operaciones aisladas. Así, una válvula hidráulica unidireccional puede ser como una válvula aórtica y la corriente sangufnea a través de la válvula puede ser modelada por la corriente de aire que los niños soplan a través de la válvula hidráulica. De igual forma (Carey 1985) cuando se intenta en el aula explicar los complejos procesos de asimilación, el paso a la sangre de las partes «buenas» de las sustancias ingeridas, puede ser representado por las propiedades de varios filtros mecánicos, cada uno de ellos permitiendo el paso de partículas de alimentos de mayor o menor tamaño, molidas por los niños. Pero, en todos los casos, los problemas de los niños son acerca del significado del modelo experimental; en la situación modelada, de hecho, necesitan conocer explicitamente qué parte, objeto, función, proceso de la realidad está representada por qué parte, objeto, función, proceso en el experimento. $Y$ en las situaciones de enseñanza, a menudo faltan reglas de correspondencia claras entre la realidad y los experimentos sugeridos to impuestos).

\section{MODELOS CIENTIfFICOS}

Creemos que los modelos infantiles de la realidad, a menudo, toman en cuenta sólo una dimensión interpretativa cada vez, así que utilizan sólo la forma espacial, o sólo la temporal, o la microscópica para interpretar la complejidad de los organismos vivos. Es difícil para un nifto o niña conectar o unir los diferentes modelos, o dar forma a un modelo basado en más de una dimension.

Sin embargo, sólo las conexiones reciprocas, la superimposición, la adecuación de modelos omnipresentes pueden producir una reconstrucción cognitiva de la realidad capaz de explicar sus distintas características, capaz de interactuar causalmente con, $y$ de modificar para propósitos definidos, cualquier suceder específico de cosas. Los modelos de los adultos, los modelos culturales, especialmente los modelos científicos, son de hecho reconstrucciones multidimensionales de aspectos de la reaildad; pero sólo sobre la base de las distintas dimensiones siempre presentes - puede emerger cada vez una, dominando sobre otras, privilegiando su forma específica de observar hechos y fenómenos. En otras palabras, solo la disponibilidad cultural e individual de muchas formas sincrónicas de elaborar modelos de distintas situaciones y relaciones entre situaciones, da a los seres humanos poder cognitivo y factual sobre la realidad y construye redes causales entre hechos y sucesos. Cuanto más rica la actividad de modelado, más profundo puede ser el dominio cognitivo de situaciones, capaz de analizar pautas complejas de la realidad de acuerdo con varios criterios distintos a! mismo tiempo. La destreza cognitiva de elaboración de modelos conduce al pensamiento científico a construir interpretaciones coordinadas de fenómenos interconec tados, y a representar por modelos jerarquizados, relaciones reciprocas, infuencias múltiples, regulaciones mutuas de la dinámica de los acontecimientos. Los modelos más complejos de retroalimentación surgen de controles por retroalimentación explícitamente reconocidos en procesos vitales específicos, y su generalización nos proporciona un potente método de conectar evidencias concretas de la realidad.

La tarea social (Pozo 1987) de enseñar ciencias a los ninos, consiste en estimular el desarrollo de sus simples actividades de modelado hacia otras más complejas y articuladas; la ensentanza de las ciencias en la escuela debe operar componiendo e integrando distintos tipos de actividades de elaboración de modelos, y de. sarrollando otros nuevos, con el objetivo de llevar gradualmente la ingenuidad de los modelos infantiles hacia la complejidad de los modelos científicos.

\section{DESARROLLO Y CRECIMIENTO DE LOS MODELOS INFANTILES (Carey 1985)}

Para interpretar y guiar la evolución de la multitud de modelos siempre presentes en la actividad cognitiva de 
los ninos, en conclusión, es importante, en nuestra opinión, tener en cuenta que:

1) Construir y validar modelos es siempre un proceso de «bucles», originándose a menudo de puntos de partida analógicos; progresando siempre por medio de estrechas correlaciones entre la selección y el ajuste de Ios datos relevantes; a veces acabando en un regulacion tan bien validada que hace extremadamente diffcil la posterior evolución del pensamiento.

2) Todo modelo, al no ser idéntico a su correspondiente evidencia factual, puede ser construido y aceptado como parcial; es decir, será útil sólo confrontado a otros diferentes (complementarios, superpuestos, alternativos, incompatibles... con él) necesarios para hacer plausible la explicación y posible el cambio de explicación.

3) Todo modelo comporta rasgos particulares de representación (varias representaciones alternativas, no equivalentes de un modelo, son en general posibles y útiles); sin embargo no hay identificación posible, ni correspondencia biunivoca, ni prioridad jerárquica, entre modelo y representación, sino una red de conexiones dinámicas.

4) Los ingredientes básicos de la construcción de modelos (impacto físico y cultural) son esencialmente similares para todo el mundo; sin embargo, incluso diferencias muy pequeñas en las estructuras de pensamiento y experiencia son capaces de producir variedades muy diferenciadas y redes de modelos «espontáneos", debido a la estructura fuertemente resonante y recurrente de la dinámica cognitiva implicada.

\section{REFERENCIAS BIBLIOGRAFICAS}

ARCA, M., GUIDONI, P., MAZZOLI, P., 1984. Structures of understanding at the root of Science Education. Part 1. Experience, language and knowledge. Part 2. Meanings for formalisation, European Journal Science Education, Vol. 5(4), pp. 367-375 y Vol. 6(4), pp. 311-319.

ARCÀ, M., FERRARINI, M., GARUTI, N., GUIDONI, P., GUERZONI, D., MAGNI, M., 1988. Esperienze sulla luce. (Petrini: Torino).

BACHELARD, G., 1979. Quelques aspects historiques des notions de modele et de justification des modeles, Actes du Colloque Elaboration et justification des modeles, pp. 9-21.

BAREITER, C., 1985. Towards a solution of the learning paradox. Review of Education Research. Vol. 55(2), pp. 201-226.
5) Aunque algunos de los modelos infantiles pueden aparecer a primera vista como muy directos y simpies, o como muy toscos, no obstante, el aparato interpretativo de los adultos, capaz de entender lo que significan en realidad e interaccionar con ellos, por fuerza debe ser muy sensible y sofisticado: incluso el más ingenuo de los modelos es en realidad la emergencia de actividades cognitivas extremadamente complejas. A veces un modelo infantil de hechos o procesos puede corresponder a una situación particular, experimentada directamente sólo una vez en la vi. da; o puede representar una sintesis de muchos acontecimientos distintos reunidos bajo un único esquemas...; o puede estar construido sobre evocaciones verbales, sobre figuras dibujadas, sobre explicaciones de adultos interpretadas estrambóticamente... y así sucesivamente. Y nuestra experiencia escolar está llena de intentos fallidos de interpretar superficialmente y «enderezar" de modo sumario, la forma en que un niño ve su mundo.

6) Un modelo global adecuado de nuestras actividades cognitivas (humanas) de construcción de modelos, está, por ahora, bastante lejos de nuestro alcance; por tanto necesitamos muchos modelos parciales, particulares, de lo que sucede en nuestras cabezas cuando construímos un modelo, con objeto de hacer progresar nuestra comprensión y de llegar a ser progresivamente más conscientes de las múltiples estrategias que usamos en nuestra búsqueda continua de una reconstrucción racional de la realidad.
CAREY, S., 1985. Conceptual change in Childhood. A Bradford book. (The MIT Press: Cambridge Massachusets).

GIORDAN, A., 1987. Los conceptos de biología adquiridos en el proceso de aprendizaje, Ensenanza de las Ciencias, Vol. 5(2), pp. 105-111.

GIORDAN, A. et al., 1983. L'elève et/ou les connaissances scientifiques. (P. Lang: Berna).

GUIDONI, P., 1985. On natural thinking. European Journal Science Education. Vol. 7(2), pp. 133-140.

MAZZOLI, P., 1987. Strategies de représentation et modèle d'interpretation a l'école primaire, Actes IX Journés Internationales sur l'Education Scientifique, Chamonix.

POZO, J.I., 1987. Aprendizaje de la ciencia y pensamiento causal, (cap. 97). (Visor: Madrid).

RIMONDI, A., 1988. Terre metalli e sole. (Petrini: Torino). 